\title{
INTERACCIÓN DE PROCESOS GEOMÓRFICOS Y DISTRIBUCIÓN DE COMPONENTES DE LA SUPERFICIE DEL SUELO EN RELACIÓN A LA EVOLUCIÓN DE LOS ABARRANCAMIENTOS DE TABERNAS (ALMERÍA)
}

\author{
A. CALVO-CASES ${ }^{(1)}$, R.W. ALEXANDER ${ }^{(2)}$, E. ARNAU-ROSALÉN ${ }^{(3)}$, J. BEVAN $^{(2)}$, \\ Y. CANTÓN ${ }^{(4)}$, R. LÁZARO ${ }^{(3)}$, J. PUIGDEFÁBREGAS ${ }^{(3)}$ y A. SOLÉ-BENET ${ }^{(3)}$
}

(1) Departament de Geografia, Universitat de València

(2) Department of Geography and Development Studies, University of Chester

(3) Estación Experimental de Zonas Áridas,

CSIC, Almería

(4) Departamento de Edafología y Química Agrícola, Universidad de Almería

correo electrónico de contacto: adolfo.calvo@uv.es

RESUMEN. Este artículo intenta analizar las interrelaciones entre la distribución a lo largo de una zona de abarrancamientos de las unidades de componentes de la superficie del suelo y los factores (topográficos, geomorfológicos e históricos) que afectan a dicha distribución. La cartografía de componentes superficiales del suelo se ha obtenido con la clasificación de una imagen multiespectral de alta resolución. Para la obtención de los parámetros topográficos, se ha construido un detallado modelo digital de elevaciones; en combinación con trabajo de campo e información previa sobre la evolución de las formas y los procesos, constituye el conjunto de datos manejados.

Los resultados muestran como la historia geomórfica y los procesos actuales interactúan y se reflejan en la distribución de componentes de la superficie del suelo.

ABSTRACT. This paper tries to analyse the relationships between the soil surface components patches distributed along a badlands area and the factors (topographical, geomorphological and historical) affecting this spatial distribution. The soil surface components maps has been obtained by a high resolution multi-spectral image classification and also a detailed digital elevation model has been made in order to obtain topographical parameters, together with field work and previous knowledge of the area on processes and landforms evolution, constitute the dataset. 
Results show how the landforms history and operating surface processes interacts and is reflected on the soil surface components distribution.

Palabras clave: Badlands, Componentes superficiales del suelo, Factores de la erosión. Key words: Badlands, Soil Surface Components, Erosion Factors.

Enviado el 15 de septiembre de 2008 Aceptado el 14 de octubre de 2008

\section{Introducción}

Los abarrancamientos, acarcavamientos, malpaís o badlands han sido descritos como zonas de intensa actividad de múltiples procesos de erosión (Harvey y Calvo, 1991; Calvo et al., 1991; Harvey, 1992) que configura un relieve intensamente disectado, con pendientes fuertes y escasa cobertura vegetal. Aunque, como consecuencia de la intensa erosión, la evolución de las formas (reducción de las pendientes, incremento de la cobertura de fragmentos rocosos) así como la formación de costras biológicas de algas y líquenes y el desarrollo de vegetación vascular, permite mecanismos de estabilización natural (Alexander et al., 1994) en una parte o en todo el conjunto del sistema.

Así, los acarcavamientos, siempre en materiales blandos propensos a elevadas tasas de meteorización y remoción, muestran, en las latitudes medias y altas, un elevado contraste derivado de la influencia de las condiciones microclimáticas en los valles de exposición contrastada, sobre las combinaciones de procesos y sus velocidades de actuación, y existen múltiples restos de antiguas laderas y fondos de valle asociados a fases de estabilización.

Se trata, por tanto de medios muy sensibles, en los que es fácil la ruptura de una situación de equilibrio ante pequeños cambios ambientales, naturales o inducidos por la actividad humana, pero que por su elevada velocidad de cambio en las formas, pueden alcanzar completa o parcialmente nuevas formas en equilibrio y con un notable descenso de las tasas de actuación de los procesos. El resultado, en un instante del tiempo, es una morfología compleja producto de múltiples cambios espacio-temporales en la combinación de procesos que actúan.

Hay, por otra parte, evidencias en la literatura de que existe una asociación significativa entre la distribución espacial de los componentes superficiales, particularmente clases de vegetación, y la de las geoformas (Alexander et al., 1994; Guàrdia, 1995; Guàrdia y Ninot, 1996) explícitamente investigada por Lázaro (1995) y Lázaro et al. (2000).

Nuestra hipótesis es que el espectro de componentes superficiales del suelo (SSC, elementos de la superficie del suelo y de cobertura que influyen sobre la actividad de los procesos; ver Arnau-Rosalén et al., 2008) de un sitio determinado contiene información sobre la historia geomorfológica del mismo, al menos a escala espacial de geoforma. 
Este artículo toma como ejemplo de esta complejidad en la evolución de geoformas y superficies la cuenca de El Cautivo (Tabernas, Almería). Integrando parte del conocimiento existente sobre ésta y el análisis numérico de las formas del relieve en una nueva cartografía de componentes superficiales del suelo, obtenida a partir de imágenes multiespectrales de alta resolución, pretende realizar un primer análisis, principalmente cualitativo y conceptual, de las relaciones entre las clases de superficie y la historia geomórfica, representada por la antigüedad de formación del sitio. Y, en función del grado de asociación entre los SSC y la antigüedad, discutir posibles detalles de dicha historia geomórfica basándonos en los componentes superficiales actuales, intentando ver si la hipótesis ha de ser rechazada. El objetivo último es establecer criterios metodológicos que pueden ser útiles para el perfeccionamiento de este análisis de relaciones y de su extensión a otras zonas del malpaís de Tabernas

\section{2. Área de Estudio}

Diferentes aspectos de la geomorfología y ecología de los abarrancamientos de Tabernas han sido estudiados, durante más de dos décadas, utilizando un amplio rango de aproximaciones y métodos. Buena parte de este trabajo se ha concentrado en la cuenca de El Cautivo donde entre otras cuestiones se ha prestado especial atención a: la evolución a largo plazo de las formas (Alexander et al., 1994 y 2008; Cantón et al., 2001a, 2003, 2004a, 2004b; Nogueras, et al., 2000), a los procesos actuales (Cantón et al., 2002; Solé-Benet et al., 1997; Lázaro et al., 2008) y la dinámica y distribución de suelos y vegetación (Cantón et al., 2001b, 2004b; Lázaro et al., 2000; Lázaro y Puigdefábregas, 1994).

La cuenca de El Cautivo (Figura 1) ocupa un sector representativo del malpaís del denominado Desierto de Tabernas. Se desarrolla sobre margas y turbiditas del Tortoniense superior con intercalaciones ocasionales de areniscas calcáreas, que en la divisoria Sur de la cuenca coronan las laderas con varios metros de espesor y dan lugar a cantiles. Estos materiales constituyen la base del relleno de la cuenca Neógena de Tabernas (Harvey, 1987). Tras el Tortoniense ha estado sometida a actividad tectónica con un proceso de levantamiento, que ha perdurado intermitentemente hasta el Cuaternario (Harvey et al., 2003). La elevación ha sido máxima en la parte Oeste de la cuenca de Tabernas, mientras que la depresión subsidente de Rioja, situada al Sur de nuestra zona de estudio, confieren a ésta una elevada ratio de relieve inducido por movimientos tectónicos.

Durante los últimos 14.000 años la zona ha registrado, al menos, cinco fases de regulación de las laderas separadas por otras tantas fases de incisión (Alexander et al., 1994 y 2008). De estas fases de estabilización quedan en la zona (Figura 1) numerosos restos de laderas o tramos de ladera (Foto 1), en ocasiones con depósitos asociados y también caracterizados por estar cubiertos por suelos más evolucionados que los de las zonas conectadas con el nivel de base actual, y tener también diferentes coberturas de vegetación (Foto 2) (Alexander et al., 1994; Lázaro, 1995; Cantón, 2001). 


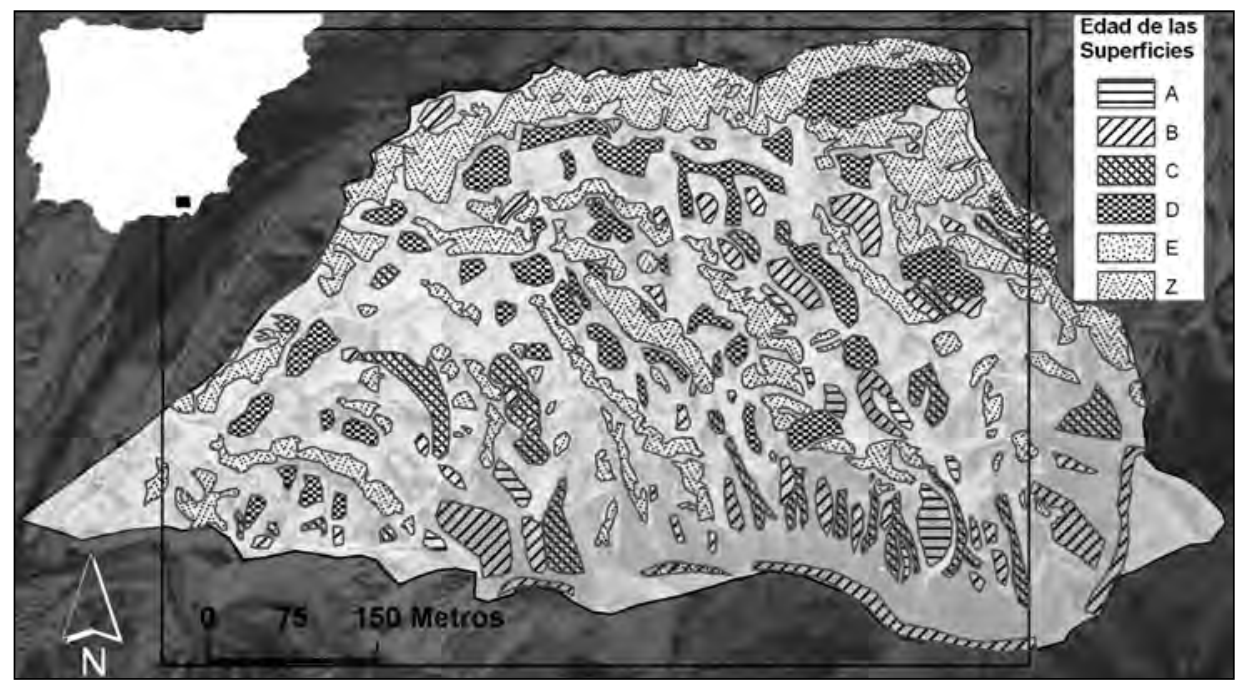

Figura 1. Mapa de localización de la cuenca de El Cautivo, con indicación de los límites de la zona de estudio determinada por la imagen ATM disponible. Superposición de la cartografía de niveles morfogenéticos (A-E) y acarcavamientos actuales (Z), según Alexander et al. (2008)

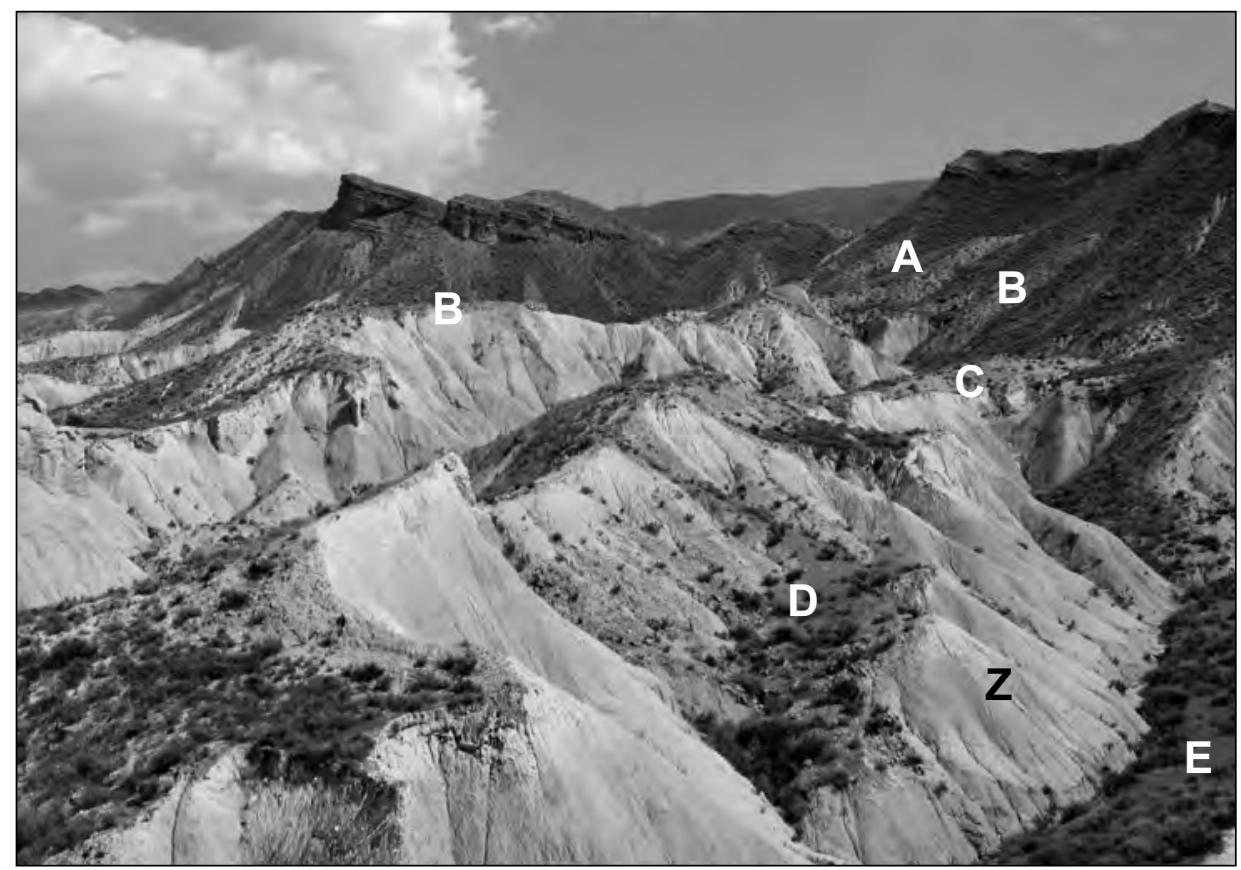

Foto 1. Vista general de las cabeceras de la cuenca donde son visibles numerosos restos de superficies correspondientes a las fases de estabilidad (algunos señalados sobre la imagen) 
Esta sucesión de fases de incisión / regularización está en relación con la actividad tectónica en la zona que, durante el Pleistoceno Superior, favoreció una ralentización de la incisión de la Rambla de Tabernas (nivel de base local de la zona de estudio) que condujo a la formación de una zona palustre. Ésta, ha sido interpretada por Harvey y Mather (1996) y Harvey et al. (1999 y 2003) como un lago o pantano denominado 'lago inferior de Tabernas' y formado a lo largo del valle principal de la Rambla de Tabernas. Su formación está en relación con la elevación de un anticlinal cuyo eje se sitúa 2,5 Km al SW de El Cautivo. Las muestras analizadas por Harvey et al. (2003) tomadas a 2,5 m del techo de esta serie palustre muestran una edad U/TH de $14 \mathrm{ka} \mathrm{(+/-} 1 \mathrm{ka})$.

El análisis geomorfológico realizado en Alexander et al. (1994 y 2008) correlaciona los niveles morfológicos de edad B (Fig. 1) con esta fase de estabilidad asociada a la formación palustre. Ello, ha permitido a Alexander et al. (2008) establecer unas tasas de denudación media desde el inicio de la incisión entre las fases $\mathrm{B}$ y C de $0,16 \mathrm{~mm}$ año ${ }^{-1}$ de descenso de suelo, equivalente a algo más de 4 millones de $\mathrm{m}^{3}$ de vaciado erosivo, entre el inicio de la disección del lago y la actualidad. Este volumen de denudación se ha producido durante tres fases de incisión, fundamentalmente ligadas al progresivo descenso del nivel de base local a medida que, primero los depósitos lacustres, y posteriormente la roca subyacente, han sido cortados por el cauce principal de la Rambla de Tabernas. Tras una primera incisión poco importante, seguida de la estabilización $\mathrm{C}$, se produjo la más importante incisión que configura la red de drenaje actual. Con estos valles ya configurados, las etapas de estabilidad D y E, caracterizadas por el desarrollo de pedimentos al pie de las laderas y una combinación de procesos que permitió el desarrollo de costras biológicas y de cobertura vegetal. Estas fases de estabilidad pueden estar relacionadas con cambios en las condiciones climáticas y/o de los usos del suelo (ver Nogueras et al., 2000).

Las laderas de las cárcavas o barrancos de la cuenca de El Cautivo muestran, como muchos otros lugares con desarrollo de acarcavamientos, diferentes grados de combinación y actividad de procesos, al tiempo que distintas densidades de cobertura, tanto de plantas como de líquenes y algas. Las tasas de infiltración final estable, medidas con simulación de lluvia, (Solé-Benet et al, 1997) abarcan un amplio rango de magnitudes (7-47 $\mathrm{mm} \mathrm{h}^{-1}$ ) y lo mismo sucede con las tasas de erosión de suelos (entre 1 y $568 \mathrm{~g} \mathrm{~m}^{-2}$ ). A escala de cuenca las tasas de erosión medias registradas en cinco años oscilan entre 100 y $450 \mathrm{~g} \mathrm{~m}^{-2} \mathrm{a}^{-1}$ (Cantón, 2001).

\section{Metodología}

Se ha elaborado una cartografía de los componentes de la superficie del suelo (SSC) con diferentes tipos de cobertura de plantas y de líquenes, pedregosidad superficial, o suelo desnudo. Este mapa (Fig. 2) ha sido realizado a partir de la clasificación supervisada de una imagen multiespectral.

La imagen fue tomada en 1996 por el NERC (Natural Environment Research Council, de Gran Bretaña, misión 95/10) con el sensor aerotransportado ATM (Airborne Thematic 
Mapper). La imagen tiene una resolución espacial de 2 m y 10 bandas de información espectral (http://arsf.nerc.ac.uk/instruments/). El mismo vuelo de NERC obtuvo fotografías aéreas analógicas estereoscópicas a color de escala grande (1:6.667). Estas fotografías se escanearon a la máxima resolución posible $(0.2 \mathrm{~m}$ por píxel $)$ y, junto con la imagen multiespectral, fueron ortocorregidas usando Erdas Imagine 8.7 Orthobase. Así mismo, usando Leica Photogrammetry Suite de Erdas 8.7, se obtuvo a partir de los pares estereoscópicos de fotografía aérea, un modelo digital de elevaciones de $1 \mathrm{~m}$ de píxel, con el cual se han calculado, usando ArcGis 9.2, los mapas de pendiente, insolación, exposición, área de contribución, red de drenaje y ordenación de esta según Strahler, así como los índices de distribución espacial (Average Nearest Neighbor y High Low Clustering) que nos indican en que medida la distribución es o no aleatoria.

Previo a la clasificación de la imagen multiespectral, se normalizó ésta, utilizando Erdas Imagine 8.5 y se aplicó un filtro de Componentes Principales (PCA). Los componentes 1,2 y 3 se tomaron como los más significativos a la hora de mostrar las diferencias en las propiedades de la superficie de los distintos lugares de la zona de estudio.

Por otra parte, se establecieron en el campo una serie de parcelas uniformes en cuanto a las propiedades de la cobertura, representativas de los principales tipos de componentes superficiales (SSC) presentes en la zona. Dichas parcelas se georeferenciaron con el uso de un GPS (Trimble GeoXT) y una vez ubicadas sobre la imagen ATM se expandió la unidad a un tamaño de varios píxeles uniformes. Usando estas zonas como referencia se realizó una clasificación supervisada de la imagen procedente del análisis de componentes principales, usando la rutina de clasificación de máxima verosimilitud con Erdas Imagine 8.5. La cartografía resultante fue comprobada en el campo y considerada aceptable, en cuanto a la representatividad de las clases de componentes superficiales y su distribución.

La historia geomorfológica de los sitos correspondientes a las diferentes manchas de la cartografía de SSC, historia cuya relación con los componentes superficiales se discute, se basa sobre todo en el mapa de edades de las superficies (Fig. 1) de Alexander et al. (1994 y 2008)

\section{Resultados}

\subsection{Cartografía de las distintas clases de superficie atendiendo a los SSC}

El mapa obtenido a partir del tratamiento y clasificación de la imagen multiespectral ATM (Fig. 2) incluye siete clases de superficies integradas por componentes superficiales del suelo (SSC) diferenciados. esas clases son, en gran medida, coincidentes con las descritas en trabajos previos basados en observaciones y mediciones en campo (Alexander et al. 1994; Lázaro, 1995; Cantón, 2001) y han sido adaptadas aquí a las limitaciones del proceso de clasificación de una imagen multiespectral de 2 m de resolución:

(1) Margas y suelo desnudo en superficie: Incluye todo tipo de superficies desnudas entre las que destacan margas 'frescas' recién erosionadas, costra mineral producida 


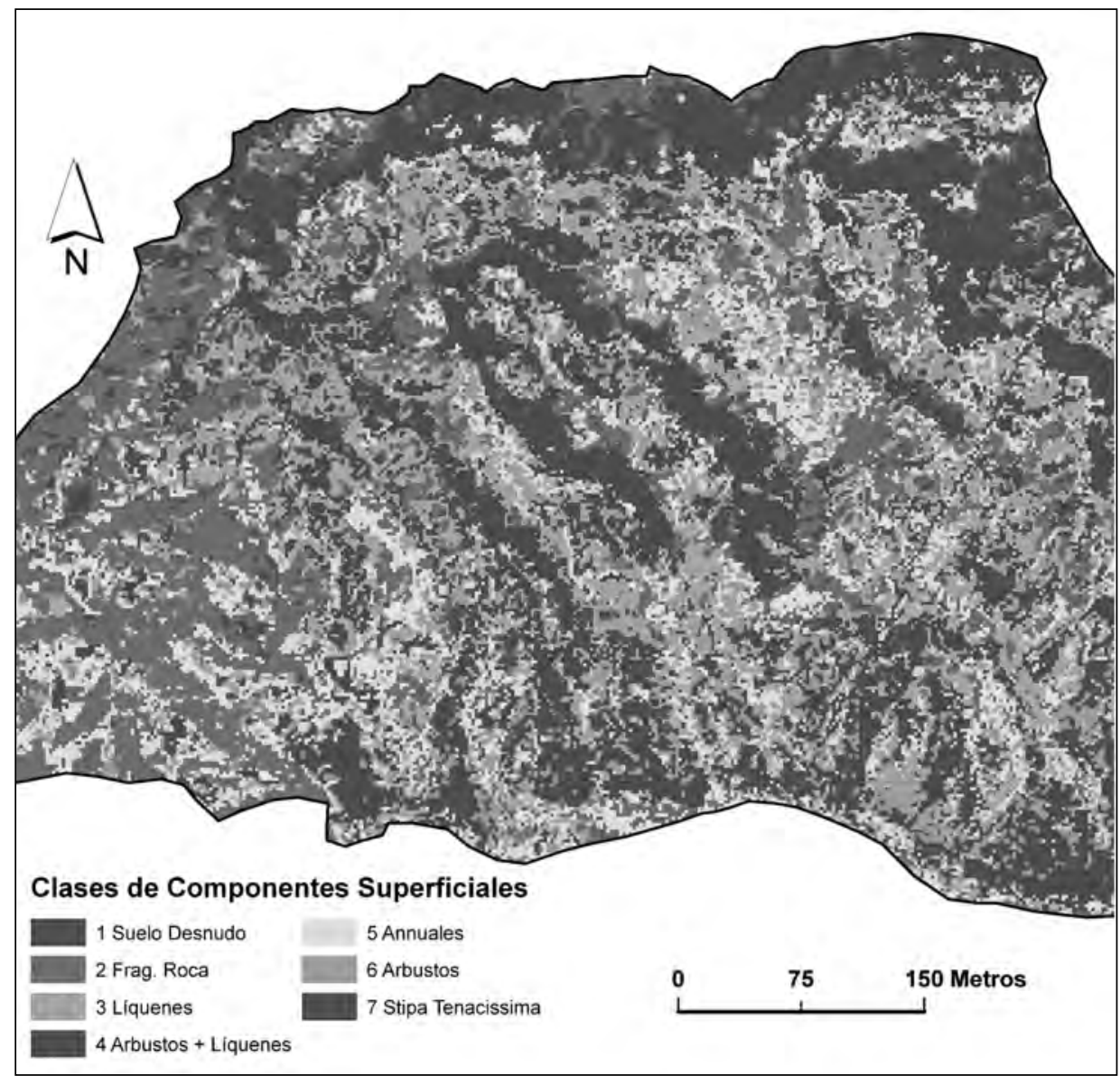

Figura 2. Mapa de componentes superficiales del suelo obtenidos tras la clasificación de la imagen ATM

por el impacto de las gotas de lluvia y, en ocasiones y en pequeñas proporciones que escapan a la escala de la imagen, suelo más evolucionado, pero desnudo, preservado de la erosión por su posición topográfica en divisorias o por una costra biológica fragmentada.

(2) Suelo desnudo con importante cubierta de fragmentos de roca o con una costra biológica 'marrón' dominada por cianobacterias (incluye también algas, hongos y diversas especies de líquenes colonizadores): En la parte Sur y SE de la cuenca de El Cautivo, los afloramientos de areniscas que coronan o se intercalan con las margas de la serie Tortoniense, aportan, desde su posición en cantiles, abundantes fragmentos de roca remanentes en superficie. Así mismo, esta clase incluye superficies de suelo cubierto por una costra biológica de color pardo-negruzco junto a la que son frecuentes fragmentos de arenisca, presente en numerosos lugares de las divisorias de aguas en las que la vegetación ha 
sido degradada por usos del suelo, pero en las que por su morfología, los procesos de erosión son más lentos y han permitido el desarrollo de dicha costra (ver Lázaro et al. 2008).

(3) Costra de líquenes predominantemente blancos: Superficies, normalmente en laderas protegidas de la incisión basal, cubiertas por una abundante y continua costra de líquenes de colores claros (Squamarina lentigera, Diploschistes diacapsis, Buellia sps. y Fulgensia sps, fundamentalmente) (ver Lázaro et al. 2008).

(4) Arbustos dispersos y costra de líquenes en los claros: En posiciones topográficas semejante a la anterior clase, en general, es la misma clase que SSC3, pero donde la costra liquénica está salpicada de arbustos pequeños (Lepraria crassissima y Squamari$n a$ sps., entre otros, suelen ser líquenes dominantes y, entre los arbustos, destacan Euzomodendron bourgeanum Helianthemum almeriense, Artemisia barrelieri, Fagonia cretica y Herniaria fontanesii).

(5) Plantas anuales: formada por pequeñas praderas de herbáceas en lugares con buena disponibilidad de agua al pie de las laderas de las clases 3 y 4 en pequeños pedimentos. La flora de anuales representa algo más de la mitad de la total, aunque la diversidad no es muy alta por fenómenos de dominancia. Las especies más frecuentes son Stipa capensis, Plantago ovata, Bromus rubens, Linum strictum, Diplotaxis virgata, Asteriscus aquaticus, Reichardia sps.

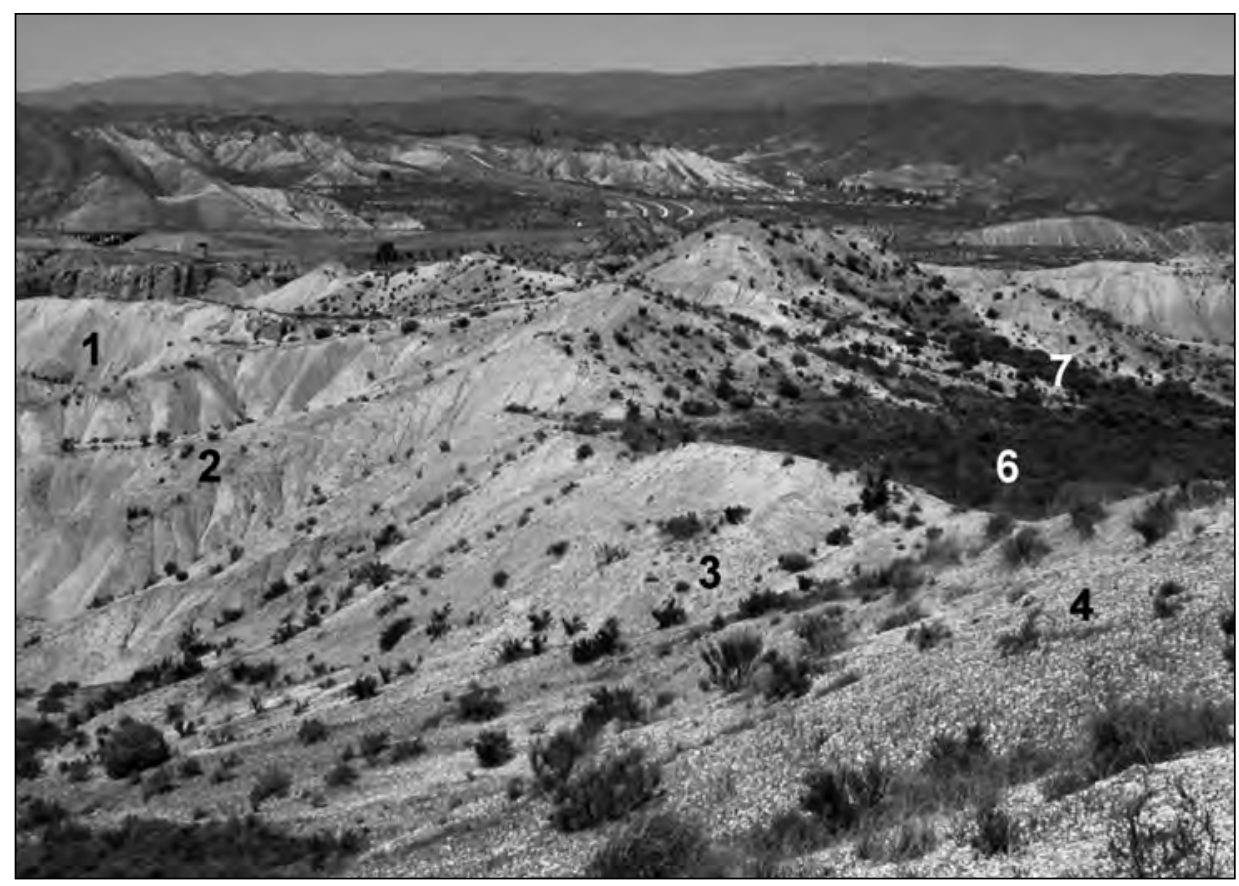

Foto 2. Una de las divisorias entre subcuencas donde conviven la mayor parte de los SSC (ver números sobre la imagen) 
(6) Cobertura densa de arbustos pequeños: En posición semejante a las clases SSC4 y 5, alcanzando los arbustos mayor densidad que en la clase SSC4, suficiente para inhibir o reducir mucho la formación de costras de líquenes. Tiende a segregarse con los prados de anuales, formando mosaicos, aunque también hay anuales entre los arbustos. Especies típicas y frecuentes son Helianthemum almeriense, Hamada articulata, Salsola genistoides, Salsola papillosa, Genista umbellata, Anabasis articulata, Anthyllis terniflora, Launaea lanifera, Artemisia barrelieri, Frankenia corymbosa y Lygeum spartum.

(7) Espartal o mezclas de espartal y matorral de arbustos pequeños: Presente en las superficies estabilizadas hace más tiempo, con buenas condiciones hídricas. Representa la última etapa de la sucesión en la vegetación presente y es la que cubre las cabeceras de la cuenca, por encima de las incisiones de la actual red de drenaje, y muchas de las áreas circundantes. En la cuenca estudiada normalmente está asociada con paleosuperficies desconectadas del sistema erosivo de la incisión actual, es donde existe una mayor densidad de vegetación y buen desarrollo edáfico. Dominan las macollas de herbáceas perennes, en particular el esparto (Stipa tenacissima), siendo también frecuentes y típicas Dactylis glometa ssp hispanica, Brachypodium retusum, Helianthemum almeriense, Hamada articulata Thymus hyemalis, Asparagus horridus, Stipa parviflora, Sideritis pusilla y Phagnalon rupestre.

\subsection{Análisis de los factores de distribución de los componentes superficiales}

La distribución de los tamaños de las distintas unidades de cada clase y su proporción del área total (Tabla 1), muestran el predominio de la clase 1 (Suelo desnudo) en ambos parámetros. Esta clase supone el $21.5 \%$ de la superficie así como los polígonos de mayor tamaño, es decir que cuenta con la mayor continuidad espacial. Ésta es una característica común en las zonas de acarcavamientos. No obstante, ello implica que casi el $80 \%$ de la superficie de estudio no corresponde a suelo desnudo, sino que está protegido por elementos tanto bióticos como abióticos. Aún más, excluyendo la clase 2 por considerarla cobertura fundamentalmente abiótica, el 59.6\% del suelo de la cuenca está cubierto por elementos bióticos, entre ellos destacan fundamentalmente los líquenes. Así, si al $20.4 \%$ de la clase 3 (Líquenes) añadimos una parte de las clases 4 y 2, el valor de la cobertura de líquenes estaría próximo al 37\% contabilizado por Cantón (2001) en una subcuenca de nuestra zona de estudio.

Otro tercio de la cuenca está ocupado por espacios cubiertos por plantas perennes $\mathrm{y}$ anuales, distribuidas fundamentalmente en las superficies antiguas (Ver Alexander et al., 1994 y 2008) y en los lugares con mejores condiciones hídricas y menor agresión de la erosión basal de las laderas. En conjunto, encontramos que la cuenca de estudio tiene tres grandes tipos de componentes superficiales distribuidos a partes iguales: por lo que las zonas de suelo desnudo quedan muy restringidas respecto al concepto clásico de acarcavamientos, mientras que más de la mitad de la superficie está integrada por componentes bióticos. Pero este $60 \%$ de cobertura de componentes bióticos es a escala de cuenca. A escalas finas, dentro de manchas de las clases SSC5 a 7, la cobertura de la 
Tabla 1. Distribución espacial y características de las unidades formadas por las diferentes clases de componentes superficiales

\begin{tabular}{|c|c|c|c|c|}
\hline \multirow{2}{*}{ Clase } & \multirow{2}{*}{ \% Área } & \multicolumn{3}{|c|}{ Tamaño Polígonos (m $\left.{ }^{2}\right)$} \\
\cline { 2 - 5 } $\mathbf{1}$ & 21.5 & 74.4 & 717.7 & 16876 \\
$\mathbf{2}$ & 18.8 & 39.9 & 424.9 & 13576 \\
$\mathbf{3}$ & 20.4 & 25.7 & 94.3 & 1992 \\
$\mathbf{4}$ & 6.7 & 12.0 & 23.5 & 364 \\
$\mathbf{5}$ & 14.4 & 16.6 & 50.5 & 1260 \\
$\mathbf{6}$ & 7.9 & 15.1 & 38.8 & 864 \\
$\mathbf{7}$ & 10.3 & 31.0 & 150.0 & 2932 \\
\hline
\end{tabular}

vegetación rara vez sobrepasa el 50\% salvo en hondonadas o pequeños microclimas. La cobertura de líquenes dentro de las clases SSC3 y 4 tampoco es total, oscilando entre el $50 \mathrm{y}$ el $100 \%$.

Atendiendo al tamaño de los polígonos como un indicador reflejo de la continuidad espacial de cada clase, el tamaño medio de los polígonos es mayor, aunque también más irregular, en las clases SSC1 y 2, es decir, las superficies más degradadas tienen un mayor grado de continuidad espacial. Siguen en tamaño los polígonos de la clase SSC7, debido a que la mayor parte de las manchas de espartal se mantienen en posiciones topográficas fuera del alcance de la incisión erosiva actual. El resto de SSCs aparecen mucho más fragmentados siendo las formaciones de líquenes blancos (SSC3) las de mayores dimensiones.

En términos de distribución espacial, los dos índices aplicados al mapa de la Fig. 2 (Average Nearest Neighbor y High Low Clustering) indican que está distribución no es aleatoria sino que esta configurada con un alto nivel de agrupamiento espacial. Factores tales como la historia geomórfica de la cuenca y la exposición contrastada de los valles explican esta tendencia al agrupamiento de las unidades de SSC.

\subsection{Correspondencia entre las clases de superficies y la evolución geomórfica}

Tal como se destaca superpuesto en el mapa de la Fig. 1, durante el proceso de evolución de la cuenca se han sucedido diferentes fases de estabilización de las formas, seguidas de fases de incisión y formación de acarcavamientos. El mapa muestra los diferentes restos de superficies correspondientes a 5 fases de estabilización denominadas de A a E en Alexander et al. (1994 y 2008). Se ha llamado Z a las laderas y cauces actualmente activos en incisión, mientras que las zonas no cartografiadas corresponden a superficies intermedias entre las fases de estabilidad / incisión. 
Al analizar los componentes superficiales presentes sobre cada una de estas paleo superficies y la actual $(Z)$ se encuentra una muy clara correspondencia entre éstas (Tabla 2). Las superficies más antiguas (A y B), con más de 14000 años de edad (ver Alexander et al., 2008) muestran un predominio de componentes de las clases 2 y 5 , así como de 6 y 7 . Son, en general, superficies vegetadas o con líquenes y piedras en el caso de los retazos que han quedado como cumbres o divisorias con escaso aporte hídrico. En las superficies $\mathrm{C}$, cuya edad es ligeramente más reciente, pero que ocupan posiciones con mejor aporte hídrico (vaguadas y largos pedimentos colgados), dominan los componentes 7, 6 y 5, los que implican mayor desarrollo de la vegetación. Entre las superficies D y E, ya situadas dentro de los valles actuales, existe una notable diferencia, por razones topográficas: En las D dominan los líquenes de la clase 3 y las acumulaciones de fragmentos rocosos, también con líquenes, de la clase 2, ubicados en superficies más degradadas en partes elevadas de las laderas. En E, dominan los arbustos (6) y las plantas anuales (5), en relación con su condición de pedimentos bien suministrados de agua de escorrentía procedente de laderas cubiertas por líquenes. Por último, las superficies de suelo desnudo (66.5\%) coinciden con las laderas de los acarcavamientos activos actualmente $(\mathrm{Z})$, normalmente conectadas con los fondos de cárcava activos que ejecutan una importante zapa basal.

Otra aproximación que nos puede indicar rangos de edad en algunos SSC es la de su distribución en relación a cauces activos actuales. A partir del modelo digital de elevaciones se ha procedido a la construcción de la red de drenaje y su ordenación según el método de Strahler. Se ha forzado la red de cauces hasta tamaños de cuenca mínimos, de modo que los cauces de orden 1 corresponden en su mayoría a vaguadas y regueros en las laderas, llegando así la cuenca a tener orden 7. Expresado como proporción de área de cada SSC, en cada orden de cauce, en una banda de $1 \mathrm{~m}$ a cada lado perpendicular al talweg, la Tabla 3 muestra (atendiendo a los valores máximos) como en los ordenes bajos se reparten las mayores proporciones los componentes SSC1 (suelo desnudo)

Tabla 2. Proporción de cada clase de SSC en las distintas superficies de la Fig. 1

(En negrita se destacan los valores más altos)

\begin{tabular}{|c|r|r|r|r|r|r|}
\hline $\begin{array}{l}\text { Clase } \\
\text { Superficie }\end{array}$ & \multicolumn{1}{|c|}{ A } & \multicolumn{1}{|c|}{ B } & \multicolumn{1}{c|}{ C } & \multicolumn{1}{c|}{ D } & E & Z \\
\hline $\mathbf{1}$ & 1.8 & 4.8 & 3.0 & 8.2 & 9.7 & $\mathbf{6 6 . 5}$ \\
$\mathbf{2}$ & $\mathbf{2 3 . 5}$ & $\mathbf{1 9 . 5}$ & 14.0 & $\mathbf{2 0 . 9}$ & 1.2 & 17.4 \\
$\mathbf{3}$ & 11.0 & 18.1 & 6.0 & $\mathbf{2 5 . 6}$ & 5.0 & 13.2 \\
$\mathbf{4}$ & 4.3 & 8.6 & 6.8 & 9.4 & 11.0 & 0.5 \\
$\mathbf{5}$ & $\mathbf{3 3 . 7}$ & $\mathbf{2 1 . 3}$ & 18.5 & 18.3 & $\mathbf{3 3 . 9}$ & 1.9 \\
$\mathbf{6}$ & 15.3 & 10.7 & $\mathbf{2 0 . 1}$ & 11.2 & $\mathbf{3 4 . 9}$ & 0.2 \\
$\mathbf{7}$ & 10.3 & 17.0 & $\mathbf{3 1 . 6}$ & 6.3 & 4.3 & 0.4 \\
\hline
\end{tabular}


Tabla 3. Proporción de cada clase de SSC en la zona situada entre los cauces y un metro a cada lado de estos, agrupados según la ordenación de cauces de Strahler (En negrita se destacan los valores más altos)

\begin{tabular}{|c|c|c|c|c|c|c|c|}
\hline Orden cauce & SSC1 & SSC2 & SSC3 & SSC4 & SSC5 & SSC6 & SSC7 \\
\hline $\mathbf{1}$ & $\mathbf{1 7 . 3}$ & 16.0 & $\mathbf{2 0 . 6}$ & 7.0 & 16.6 & 10.2 & 12.3 \\
$\mathbf{2}$ & $\mathbf{2 0 . 0}$ & 14.3 & $\mathbf{1 8 . 1}$ & 7.0 & 16.5 & 11.4 & 12.8 \\
$\mathbf{3}$ & $\mathbf{2 0 . 9}$ & 13.8 & $\mathbf{1 9 . 5}$ & 6.8 & 14.0 & 9.9 & 15.1 \\
$\mathbf{4}$ & $\mathbf{2 3 . 3}$ & 10.4 & $\mathbf{2 6 . 9}$ & 7.0 & 12.4 & 8.9 & 11.1 \\
$\mathbf{5}$ & $\mathbf{4 0 . 9}$ & 7.5 & 20.8 & 4.2 & 14.3 & 7.8 & 4.4 \\
$\mathbf{6}$ & $\mathbf{4 0 . 7}$ & 11.0 & 20.2 & 8.2 & 17.4 & 1.3 & 1.3 \\
$\mathbf{7}$ & 10.9 & $\mathbf{2 7 . 2}$ & $\mathbf{3 0 . 6}$ & 1.4 & 16.3 & 3.4 & 10.2 \\
\hline
\end{tabular}

y SSC3 (líquenes), y las proporciones mínimas en los de plantas vasculares, claramente lejos de los cauces. En los ordenes de cauce 5 y 6 (valles principales con importante incisión) existe ya un dominio de píxeles vecinos de la SSC1, y en segundo lugar de la SSC3. Quizá la información más relevante en términos evolutivos nos la da el tramo de cauce de mayor orden de la cuenca. Aquí, en un valle más ancho, los píxeles de clase SSC1 han cedido su protagonismo a los de clase SSC3 y 2, e incluso SSC5 (anuales): este tramo de cauce, más antiguo, es más ancho por la evolución de las laderas y el desarrollo de micro-pedimentos en su base con signos de estabilización por pedregosidad y líquenes o anuales.

4.4. Correspondencia entre las clases de superficies y los factores topográficos principales: orientación, pendiente, posición en la ladera

El efecto de la exposición de las laderas y, por tanto, de la radiación solar sobre la distribución de los componentes superficiales del suelo queda plasmado en la Fig. 3. Las superficies desnudas (SSC 1) están distribuidas en exposiciones entre S y W. En el desvío hacia el $\mathrm{W}$ de la exposición meridional ha influido la orientación del drenaje, en parte estructural (alineaciones SW-NE), en parte obligado a virar hacia el SW por la historia evolutiva de la cuenca que incluye una importante captura de la parte norte de la cuenca por parte de la más meridional y que ocurrió durante la fase de incisión posterior a la superficie C (ver Alexander et al., 2008).

Las áreas correspondientes a la clase 2 no tienen una clara orientación preferente, sólo un cierto predominio de la W, aunque si una distribución casi idéntica en las entradas de radiación solar a las laderas de la clase 1 (Fig. 3B). Esta clase está presente sobre todo en cumbres y divisorias, o en pedimentos colgados, como lo muestra la abundancia de superficies de pendiente inferior a 20 grados (Fig. 3A). 
Las superficies con líquenes (clase 3) ocupan todo el rango de exposiciones como efecto de su desarrollo también en las cumbres, pero predominan en las laderas entre el NW y NE, siendo la exposición un factor más relevante cuando la pendiente se sitúa entre 20 y 50 grados (Fig. 3).

Las plantas perennes (clases 4, 6 y 7) son prácticamente exclusivas de las áreas expuestas entre NW y NE y con pendientes superiores a 10 grados, lo que puede expli-

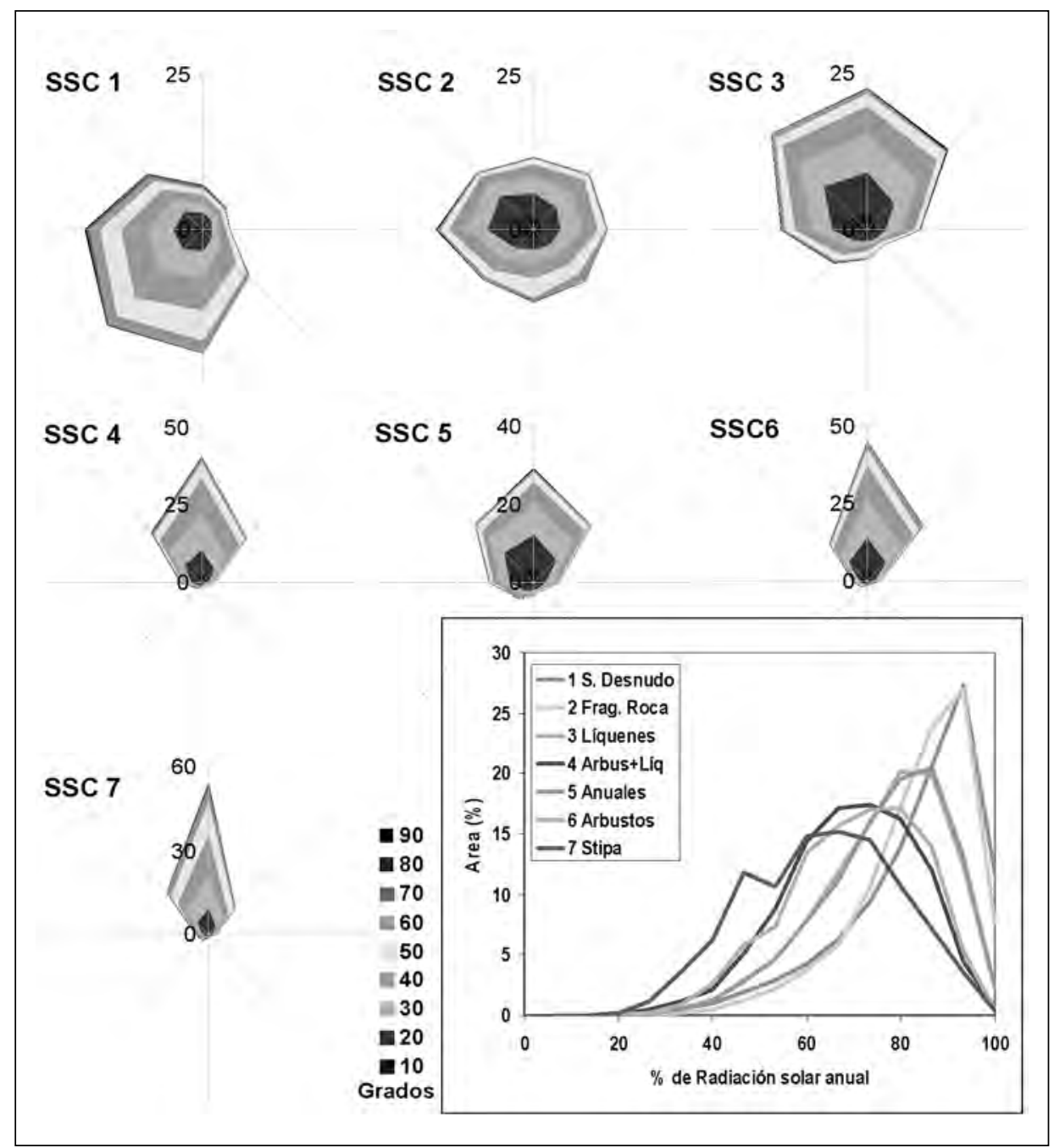

Figura 3. Frecuencia de exposición de cada una de las clases de SSC con indicación de la pendiente del píxel. Encuadre: Distribución proporcional del área ocupada por los píxeles de cada clase de SSC en función de la proporción de radiación solar a cielo despejado recibida por el píxel 
carse en relación con la necesidad tanto de una exposición protegida de la radiación (Fig. 3B) como de un cierto aporte de agua de escorrentía. Por el contrario las plantas anuales (clase 5), aunque también centradas en las laderas entre NW y NE, aparecen en otras exposiciones, incluso más al $\mathrm{W}$, y en todo el rango de pendientes inferiores a 50 grados.

La Fig. 3B, más sensible a mostrar las diferencias de la influencia de la exposición al considerar en el cálculo de la radiación el efecto de la pendiente y las sombras, muestra los SSC en 4 grupos. Los componentes 1 y 2 en las zonas más expuestas a la radiación; los líquenes y anuales (componentes 3 y 5) en mejores condiciones, pero aún muy expuestos; y los componentes que incluyen vegetación perenne en los espacios más protegidos, especialmente el esparto, aunque, en este caso, diferenciado porque ocupa los espacios de mayor pendiente al pie de los cantiles del borde Sur de la cuenca.

Junto al factor exposición aparece reflejada en la cartografía cierta influencia de la posición en la ladera ligada a las disponibilidades de agua para las plantas aparece reflejada en la cartografía. Atendiendo a la relación entre el área contribuyente y la superficie total de cada clase en cada valor de acumulación de flujo (Fig. 4A), destaca que las clases con plantas vasculares perennes aparecen en menor media en las divisorias, mientras que las de suelo desnudo y con cubierta de líquenes no muestran estas restricciones hídricas. Igualmente, atendiendo a la distribución de las clases por altitud (Fig. 4B) los SSC con cobertura vegetal vascular son más frecuentes en el rango elevado de la cuenca, mientras que superficies degradas aparecen en las zonas más bajas.

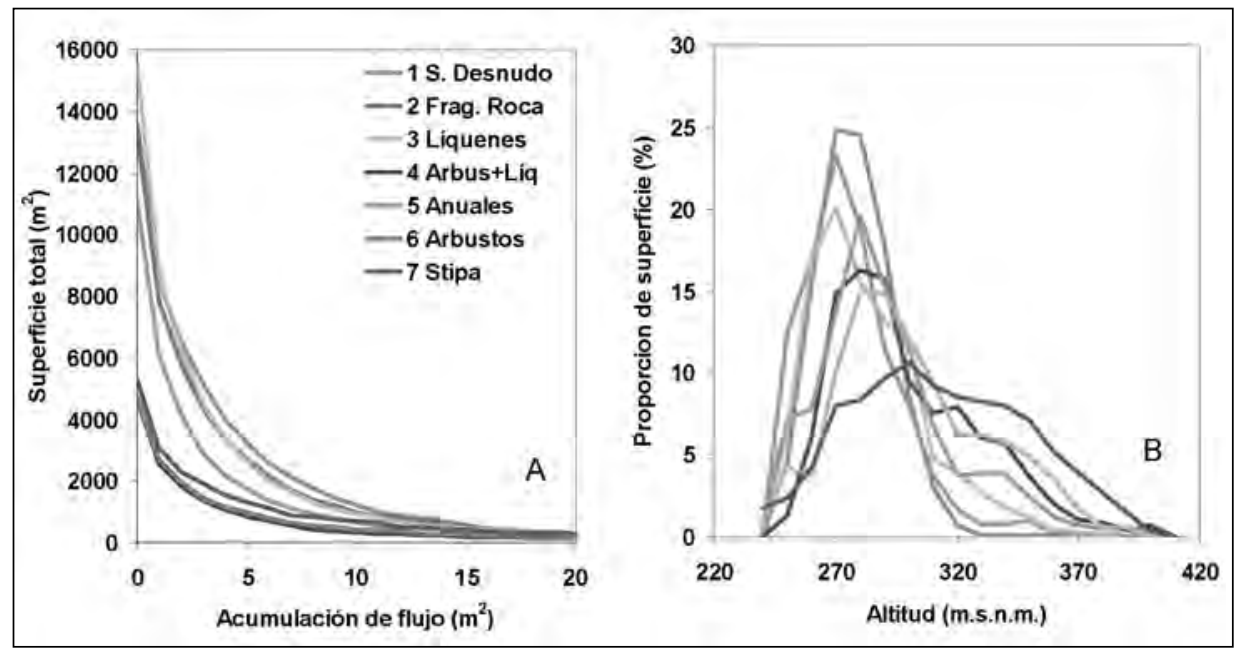

Figura 4. Relaciones entre las clases de SSC y la posición en la cuenca expresadas como: (A) Superficie ocupada por cada clase de SSC en función de los valores del área contribuyente en escorrentía a cada píxel de hasta $20 \mathrm{~m}^{2}$. (B) Proporción del área de cada SSC en relación a la altitud absoluta del los píxeles 


\section{Discusión y Conclusiones}

Los acarcavamientos o malpaís como las aquí analizadas muestran una dinámica compleja caracterizada por la presencia durante su evolución y actividad actual de múltiples interacciones de procesos de erosión y de los factores que afectan a estos procesos. Esta complejidad dista de los conceptos clásicos en geomorfología que asocian los acarcavamientos a espacios de fuerte disección lineal comandada por procesos de arroyada superficial. El resultado de la compleja interacción espacio-temporal, y al mismo tiempo la causa de ésta, es un sistema en el que conviven diversos tipos de suelo y componentes superficiales de éstos, sobre formas topográficas de pendientes variables.

En un contexto de fuerte ratio de relieve como consecuencia de la actividad tectónica y con importantes oscilaciones en el nivel de base local de la cuenca, que es la Rambla de Tabernas (Alexander et al., 2008), la evolución de los cauces y laderas ha estado afectada por el trabajo de la erosión remontante. De modo que las partes más elevadas de las cabeceras y divisorias entre valles de cárcavas conservan numerosos vestigios de formas del relieve antiguas y, por tanto, suelos más lavados, más potentes y con mejores condiciones hídricas (Alexander et al., 1994; Cantón, 2001), mientras que, por el contrario, las laderas conectadas con los valles actuales, las expuestas entre S y W, están afectadas por intensos procesos de erosión que incluyen, además de salpicadura y arroyada difusa, un importante y sostenido desarrollo de regueros (Calvo y Harvey, 1996) que alterna, en ocasiones y dependiendo del espesor que alcanza la regolita, con coladas de barro.

Analizando esta interacción en términos de equilibrio, la dinámica de las formas y procesos activos en el malpaís de El Cautivo durante su evolución y en la actualidad nos muestran una situación de 'equilibrio dinámico meta-estable' (ver Chorley y Kennedy, 1971), es decir, una evolución con fluctuaciones dentro de un proceso de cambio progresivo hacia la búsqueda de un equilibrio, que se va reflejando en formas regularizadas y el desarrollo de suelos y de componentes superficiales distintos a la roca o regolito desnudos. Pero, en determinados momentos, dentro de este espacio temporal, se ha producido la llegada a la cuenca de 'ondas de agresión' (Brunsden, 2001), expresadas por el retroceso de la cabecera de cárcavas desde los cauces principales a los tributarios, que ha determinado importantes repercusiones en la actividad de los procesos en las laderas, resultando en cambios en las formas y de los SSCs e implicando el reinicio de una nueva fase evolutiva.

La secuencia de cambios, relacionada con umbrales extrínsecos o intrínsecos, muestra en la cuenca de El Cautivo que los cambios introducidos por un importante proceso de descenso del nivel de base local en algún momento posterior a $14.000 \mathrm{BP}$, no han permitido el retorno de las formas a la situación de partida. El sistema se ha compartimentado incrementando la densidad de drenaje. Dentro de esos valles la evolución de las formas ha llevado en varias ocasiones a alcanzar situaciones más estables, pero nunca durante un lapso de tiempo suficiente para alcanzar un desarrollo de suelo y SSC similar a las fases de estabilidad más antiguas. Estas fases de estabilidad recientes han sido interrumpidas por umbrales de cambio $\mathrm{y}$, en respuesta, se han generado nuevas 
áreas de suelo y roca desnudos, con un importante incremento de la energía disponible para la actividad de los procesos, como consecuencia de la remoción de los suelos protegidos líquenes y vegetación y del incremento de la pendiente.

La mayor proporción de superficies en la clase 1, la mayor continuidad de los polígonos de esta clase, así como la proporción de área de esta clase junto a los cauces de orden medio, muestran que el proceso de incisión es reciente y sigue activo en la zapa basal de las laderas en los valles de menor orden, donde conviven a un lado suelo desnudo y en el opuesto cubierta de líquenes y de anuales, donde hay más aporte hídrico en pedimentos colgados.

Por otro lado, en el cauce principal, ha ocurrido un desarrollo de laderas y formación de micro-pedimentos a su pie en los que aparece el SSC 2 (pedregosidad y costra marrón) el componente primer colonizador de zonas inicialmente estabilizadas, que es a su vez el dominante en la parte occidental de la cuenca. Esto nos indica un desacoplamiento o desconexión entre las laderas y el cauce (Brunsden, 2001; Faulkner, 2008) tras el ascenso hacia los valles tributarios de una de las ondas de agresión de la última fase de incisión. En la estabilidad de las partes bajas ha podido tener un papel relevante el mantenimiento durante las últimas décadas de campos de cultivo a la salida de la cuenca, en la terraza baja de la Rambla de Tabernas, unos dos metros sobre el cauce que han producido un funcionamiento desconectado del nivel de base de la rambla durante décadas. Recientemente una nueva onda de agresión se está produciendo una cárcava que se activó en 2004 y más intensamente en 2005 está disectando esta terraza; ha remontado notablemente pero aún no ha afectado al interior de la zona de estudio.

La situación actual es de progresión de la incisión de los cauces y degradación de las laderas. Pero la erosión sólo parece afectar de manera significativa a una parte del área. La abundancia de otros SSC asociados a estabilidad en más de la mitad de la superficie total, muestra que durante estos casi quince milenios las ondas de agresión no han llegado a repercutir sobre todo el sistema, o al menos no las más recientes, pues han sido interrumpidas y se han iniciado procesos de estabilización llegando a situaciones de quasi-equlibrium (Alexander et al., 1994; Lázaro et al., 2008).

Esta sucesión de fases evolutivas con equilibrios y desequilibrios, junto con el hecho de que el grado de equilibrio haya sido variable y siga siéndolo en distintos puntos del espacio ha producido una dinámica geomorfológica a diferentes velocidades según los puntos y ha configurado en los abarrancamientos de El Cautivo una morfología compleja que, como suele ocurrir en este tipo de ambientes, como consecuencia de la relativa rapidez en los cambios morfológicos en relación con relieves desarrollados sobre otros materiales, permite la pervivencia de elementos de las formas tales como los suelos y la cobertura vegetal, que son parte del esquema de componentes superficiales (SSC), pervivencia que se reflejan en el medio actual. Ello conduce a una interacción espacial compleja con la actuación de conjuntos de procesos diferentes en laderas y valles que se encuentran a poca distancia entre sí. Y sugiere además interacciones entre procesos que ocurren a distintas escalas temporales. 
Así, las cabeceras de la cuenca y algunas divisorias entre subcuencas están ocupadas por componentes de mayor capacidad de infiltración y baja erodibilidad (SSC 6 y 7) principalmente; de ese modo, el tamaño efectivo de las cuencas ante eventos de precipitación está aquí reducido. El resultado de las fases de estabilidad más recientes (D y E) está presente en la cuenca como laderas regularizadas cubiertas principalmente de líquenes (SSC 3) y también líquenes más pedregosidad (SSC 2) que son enormemente protectoras del suelo y estables (Lázaro et al., 2008) en términos de movilización de sedimento (transporte limitado) pero que son contribuyentes netos de abundante escorrentía al cauce (Alexander y Calvo, 1990), no obstante, cuando la ladera de exposición Norte presenta a su base un pedimento en el que se desarrollan plantas anuales o arbustos (SSC 4, 5 y 6) el aporte al cauce es muy reducido (Cantón et al., 2002).

La pervivencia de SSC integrados por plantas y costras biológicas, asociada a los factores micro-ambientales derivados de la exposición a la radiación solar, ha contribuido a la marcada asimetría de los valles de la parte media de la cuenca, donde el efecto de las ondas de agresión actual y subactuales es más evidente, tanto por los cauces vacíos de todo sedimento, como por las laderas de suelo desnudo completamente conectadas a los cauces. Por el contrario las laderas protegidas por los SSC 2, 3, 5 y 6 terminan en pedimentos elevados respecto de los cauces actuales. Sin embargo, el efecto de las ondas de agresión se refleja también en éstas laderas estables porque están surcadas por algunos regueros longitudinales a las laderas; es por esta fragmentación que las manchas de los SSC 3, 4 y 5 tienen tamaños medios pequeños.

Junto a esta interacción espacial de procesos debida a las alternancias en la dinámica de la cuenca y los restos de formas y SSC que han pervivido, ya dentro de las áreas de la clase SSC 1, la más característica de los paisajes de abarrancamientos o malpaís y la de mayor sensibilidad de respuesta a los cambios, se produce otra interacción de procesos en gran medida controlada por los ciclos de meteorización que generan mayor o menor cantidad de material disponible para ser removido y transportado cuando se produzca un evento de lluvia (control por meteorización). El resultado es una dinámica espacio-temporal variable.

Nuestros resultados sugieren que las relaciones entre las clases de superficie y la historia geomorfológica del sitio correspondiente merecen ser estudiadas y el objetivo final de establecer criterios metodológicos para este análisis y su extensión a otras áreas se refuerza. Aunque cabe mejorar la cartografía a partir de imágenes multiespectrales, en particular para detectar mucho mejor algunos SSC de grano fino como las costras biológicas. La posibilidad de usar imágenes hiperespectrales y técnicas espectroscópicas para detectar incluso costras vivas de colores parecidos al de la superficie del suelo o las piedras debe ser explorada.

Nuestra hipótesis inicial no puede ser rechazada, siendo de momento útil para continuar avanzando, aunque probablemente pueda mejorarse su enunciado, por ejemplo precisando en qué clase de componentes superficiales está sobre todo la información acerca de la historia geomórfica. 
Las áreas de abarrancamientos o malpaís se confirman como idóneas para estudiar las relaciones dinámicas entre la geomorfología y los SSC, tanto por el pequeño tamaño de las geoformas, como por su rápida dinámica.

\section{Agradecimientos}

Este trabajo se ha desarrollado en el contexto de los proyectos: REN2003-04570/GLO (PECOS); CGL2007-63258/BOS (PREVEA) y CGL2006-11619 (PROBASE), financiados por la CICYT en el marco del Plan Nacional I+D+I. También agradecemos a la misión 95/10 del NERC (Natural Environment Research Council, de Gran Bretaña) por las imágenes ATM y las fotografías aéreas.

\section{Referencias bibliográficas}

Alexander, R.W., CAlvo-CASES, A. (1990). The influence of lichens on slope processes in some Spanish badlands. En: Vegetation and Erosion (Thornes, J.B., Ed.), Wiley, Chichester, 385-398.

Alexander, R.W., Harvey, A.M., Calvo, A., James, P.A., Cerda, A. (1994). Natural stabilisation mechanisms on Badland Slopes: Tabernas, Almería, Spain. In Environmental Change in Drylands (Millington, A.C. and Pye, K., Eds) Wiley, 85-111.

Alexander, R.W., Calvo, A., Arnau, E., Mather A.E., LÁzaro Suau, R. (2008). Erosion and stabilization sequences in relation to base level changes in the El Cautivo badlands, SE Spain. Geomorphology. 100: 79-86.

Arnau-Rosalén, E., Calvo-Cases, A., BoiX-Fayos, C., Lavee, H., Sarah, P. (2008). Analysis of soil surface component patterns affecting runoff generation. An example of methods applied to Mediterranean hillslopes in Alicante (Spain). Geomorphology, 101: 595-606.

BRUNSDEN, D. (2001). A critical assessment of the sensitivity concept in geomorphology. Catena, 42: 99-123.

Calvo-Cases, A., Harvey, A.M., PayÁ-Serrano, J. (1991). Process interactions and badland development in SE Spain. En Soil Erosion Studies in Spain (Sala, M., Rubio, J.L. y García-Ruiz, J.M., Eds). Geoforma Ed., 73-90.

Calvo-Cases, A., Harvey, A.M. (1996). Morphology and development of selected badlands in southeast Spain: Implications of climatic change. Earth Surf. Proc. Landf., 21, 725-735. 
CANTÓN, Y. (2001). Efectos hidrológicos y geomorfológicos de la cubierta y propiedades del suelo en paisajes de cárcavas. Tesis Doctorales de la Universidad de Almería. Servicio de Publicaciones Universidad de Almería. 390 pp.

Cantón, Y., Domingo, F., Solé-Benet, A., Puigdefábregas, J. (2001a). Hydrological and erosion response of a badlands system in semiarid SE Spain. Journal of Hydrology, 252: 65-84.

CAntón, Y., SolÉ-Benet, A., Queralt, L., Pini, R. (2001b). Weathering of a gypsumcalcareous mudstone under semi-arid environment in SE Spain: laboratory and fieldbased experimental approaches. Catena, 44: 111-132.

CAntón, Y., Domingo, F., Solé-Benet, A., Puigdefábregas, J. (2002). Influence of soil surface types on the overall runoff of the Tabernas badlands (South-East Spain): field data and model appraches. Hydrological Processes, 16: 2621-2643.

CAntón, Y., SolÉ-Benet, A., LÁZARo, R. (2003). Soil-geomorphology relations in gypsiferous materials of the Tabernas desert (Almería, SE Spain). Geoderma, 115: 193-222.

CAntón, Y., Del BArrio, G., Solé-Benet, A., LÁZAro, R. (2004a). Topographic controls on the spatial distribution of ground cover in the Tabernas badlands of SE Spain. Catena, 55: 341-365.

Cantón, Y., SolÉ-Benet, A., Domingo, F. (2004b). Temporal and spatial patterns of soil moisture in semiarid badlands of SE Spain. Journal of Hydrology, 285: 199-214.

ChORley, R.J., Kennedy, B.A. (1971). Physical Geography. A Systems Approach. Prentice-Hall. London. 370 pp.

FAULKNER, H. (2008). Connectivity as a crucial determinant of badland morphology and evolution, Geomorphology, 100: 91-103.

GUÀRDIA R. (1995). La colonitzacio vegetal de les arees erosionades de la conca de La Baells (Alt Llobregat). Doctoral Thesis, University of Barcelona.

GUÀRDIA, R., NINOT, J.M. (1996): Distribution of plant communities in the badlands of the upper Llobregat basin (southeastern Pyrenees). Studia Geobotanica, 12: 83-103.

HARVEY, A.M. (1987). Patterns of Quaternary aggradational and dissectional landform development in the Almería region, southeast Spain: a dry-region, tectonically active landscape. Die Erde, 118: 193-215.

HARVEY, A.M. (1992). Process interactions, temporal scales and the development of hillslope gully systems: Howgill Fells, northwest England. Geomorphology, 5: 323-344.

Harvey, A., Calvo-Cases, A. (1991). Process interactions and rill development on badland and gully slopes. Z. Geomorph. Suppl.-Bd., 83: 175-194. 
HARvey, A.M., MATHER, A.E. (1996). Tabernas Quaternary alluvial fans and "lake"system. En 2nd Cortijo Urra Field Meeting, Southeast Spain: Field Guide (Mather, A.E. y Stokes, M., Eds), University of Plymouth, 39-42.

HARvey, A.M., Foster, G., HANnAM, J., MATHER, A.E. (1999). Mineral magnetic characteristics of the soils and sediments of the Tabernas alluvial fan and "lake" system. En BSRG/BGRG SE Spain Field Meeting Guide Book (Mather, A.E. y Stokes, M., Eds), University of Plymouth, 43-61.

Harvey, A.M., Foster, G., Hannam, J., MAther, A.E. (2003). The Tabernas alluvial fan and lake system, southeast Spain: applications of mineral magnetic and pedogenic iron oxide analyses towards clarifying the Quaternary sediment sequences, Geomorphology, 50: 151-171.

LÁZARO SUAU, R. (1995). Relaciones entre vegetación y geomorfología en el área acarcavada del Desierto de Tabernas. Tesis Doctoral. Universidad de Valencia. 241 pp. + índices y anexos.

LÁZARo, R., Puigdefábregas, J. (1994). Distribución de la vegetación terofítica en relación con la geomorfología en áreas acarcavadas cerca de Tabernas, Almería. Monografías de flora y vegetación Béticas, 7-8: 127-154.

LÁzaro, R., Alexander, R.W., Puigdefábregas, J. (2000). Cover distribution patterns of lichens annuals and shrubs in the Tabernas Desert, Almería, Spain. In: Vegetation Mapping: From Patch to Planet (Alexander, R.W. and Millington, A.C., Eds). Wiley, Chichester, 19-39.

LÁzaro, R., Cantón, Y., Solé-Benet, A., Bevan, J., AleXander, R., SAncho, L.G., PUIGDEFÁBREGAS, J. (2008). The influence of competition between lichen colonization and erosion on the evolution of soil surfaces in the Tabernas badlands (SE Spain) and its landscape effects. Geomorphology, 102: 252-266.

Nogueras, P., Burjachs, F., Gallart, F., Puigdefábregas, J. (2000). Recent gully erosion in the El Cautivo badlands (Tabernas, SE Spain). Catena, 40: 203-215.

Solé-Benet, A., Calvo, A., Cerdá, A., Lázaro, R., Pini, R. y Barbero, J. (1997). Influences of micro-relief patterns and plant cover on runoff related processes in badlands from Tabernas (SE Spain). Catena, 31: 23-28. 